\title{
AIP $\left.\right|_{\text {Plyids of }} ^{\text {Physe of }}$
}

Theory of the Positive Column Including Electron-Ion Recombination

R. G. Lindgren and G. R. Gavalas

Citation: Physics of Fluids 13, 1499 (1970); doi: 10.1063/1.1693109

View online: http://dx.doi.org/10.1063/1.1693109

View Table of Contents: http://scitation.aip.org/content/aip/journal/pof1/13/6?ver=pdfcov

Published by the AIP Publishing

\section{Articles you may be interested in}

Electron-ion recombination and plasma field effects

AIP Conf. Proc. 322, 69 (1995); 10.1063/1.47079

Electron-ion recombination in merged beams

AIP Conf. Proc. 275, 228 (1993); 10.1063/1.43775

Resonant recombination and autoionization in electron-ion collisions

AIP Conf. Proc. 205, 418 (1990); 10.1063/1.39259

The theory of electron-ion collisions

AIP Conf. Proc. 205, 137 (1990); 10.1063/1.39205

Electron-ion recombination in water vapor

J. Chem. Phys. 80, 4564 (1984); 10.1063/1.447205

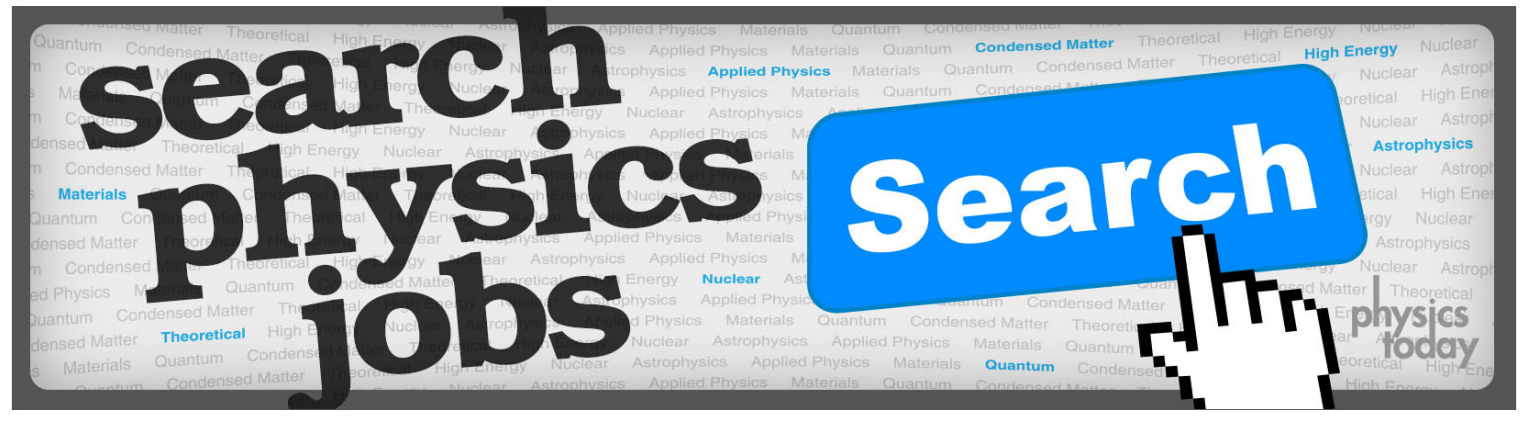




\title{
Theory of the Positive Column Including Electron-Ion Recombination
}

\author{
R. G. Lindgren and G. R. Gavalas \\ Chemical Engineering Laboratory, California Institute of Technology \\ Pasadena, California 91109
}

(Received 27 October 1969; final manuscript received 12 January 1970)

\begin{abstract}
A theoretical study of the effect of electron-ion recombination on the voltage-current characteristic of the positive column of a glow discharge is presented. In the absence of recombination, as the current increases the voltage decreases and reaches a constant value. In the presence of recombination and at sufficiently high currents, the voltage increases with increasing current.
\end{abstract}

\section{INTRODUCTION}

The voltage-current characteristic of a glow discharge, shown schematically in Fig. 1, is the sum of the characteristics of the cathode region and the positive column. The cathode region, described in many standard texts, e.g., in Von Engel, ${ }^{1}$ is qualitatively understood but due to its physical complexity has not been as yet subjected to a precise mathematical treatment. The positive column was studied by Allis and Rose ${ }^{2}$ and was recently put on a firm mathematical basis by Cohen and Kruskal ${ }^{3}$ and Blank. ${ }^{4}$ In the present paper we examine the recombination process which can significantly alter the voltage-current characteristic of the positive column in the region of high electron densities.

The theory of the positive column is based on the fact that at steady state the production of charged particles by ionization is exactly balanced by the loss by diffusion to the walls and by recombination. The axial electric field $E_{z}$ enters the working macroscopic equations indirectly through the ionization, diffusion, and various other coefficients. A relationship is then sought between $E_{z}$ and the electron density at the axis of the column $N_{00}$. This relationship implicitly specifies the voltage-current characteristic, because the voltage is determined by the axial electric field, and the current by the electron density and by the drift velocity which in turn depends on $E_{z}$.

By neglecting recombination and assuming that the ionization and diffusion coefficients do not vary in the radial direction, Cohen and Kruskal ${ }^{3}$ derive an $E_{z}-N_{\mathrm{o} 0}$ curve shown schematically in Fig. 2 . At very low electron densities $N_{\circ 0}$, the loss of electrons is by free diffusion to the wall. The working equations do not have a nontrivial solution for $E_{z}>E_{z b}$, where the bifurcation point $E_{z b}$ can be obtained from the linearized version of the equations and corresponds to breakdown. Although linearization determines the point $E_{z b}$, the $E_{z}-N_{a 0}$ curve depends on the nonlinear terms which represent

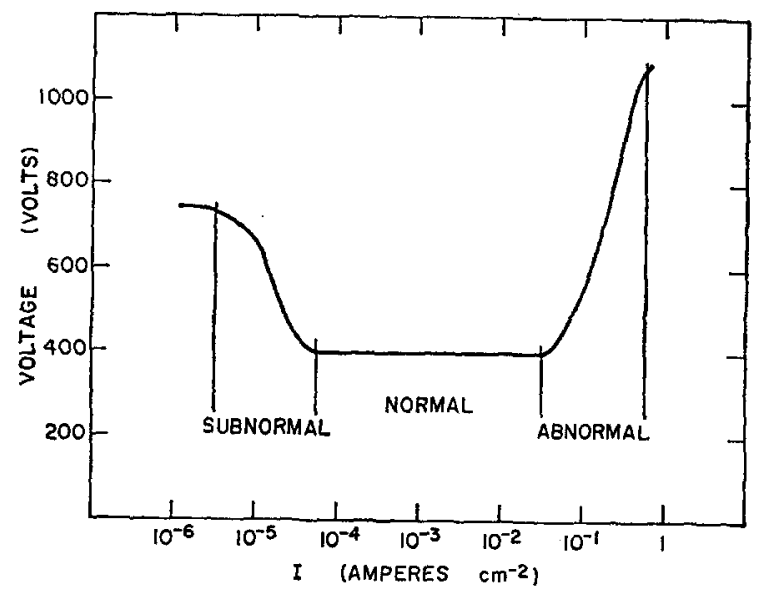

FIG. 1. Schematic representation of the voltage-current characteristic of a glow discharge.

the effect of the slight charge separation. As $N_{\bullet 0}$ increases, the radial electric field resulting from the charge separation increases and retards the diffusion of electrons while it speeds up the diffusion of positive ions. The loss by diffusion relative to the production by ionization is thereby reduced and a lower $E_{z}$, which results in a smaller ionization coefficient, is required for maintaining a steady state. In fact, as $N_{\mathrm{ol}} \rightarrow \infty, E_{z} \rightarrow E_{z a}$ which defines the limit of ambipolar diffusion, Fig. 2.

Physical considerations require that $N_{00}$ must remain finite, so before $E_{\text {z }}$ reaches the value $E_{\text {zas }}$ some other physical processes which limit the value of $N_{\text {oo }}$ must become important. In. this work we investigate one such process, the electron-ion recombination which results in an $E_{z}-N_{c 0}$ curve shown schematically in Fig. 2. At low $N_{e 0}$ the curve coincides with that of Cohen and Kruskal. Near the ambipolar limit, however, the curve bends and starts increasing with $N_{\bullet 0}$. At very high electron densities, of course, the equations for a weakly ionized gas cease to apply. The abnormal glow characteristic has so far $^{1,5}$ been attributed entirely to electrode effects. The results of this work, shown schematically 


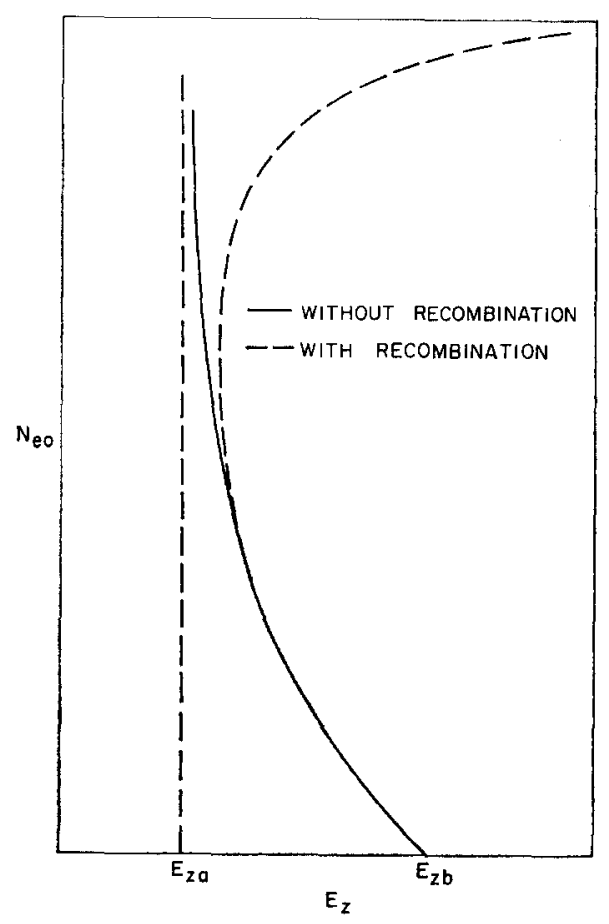

FIG. 2. Schematic representation of $E_{z}-N_{e 0}$ curves.

in Fig. 2, indicate the possibility that electron-ion recombination in the positive column may also be important in determining the over-all characteristic in the abnormal glow region.

As recombination can only be important at high electron densities, it is there that we focus our attention. In this case we use ambipolar diffusion and thus avoid treating space-charge effects explicitly. This approach is consistent with a detailed analysis which we have carried out treating spacecharge effects and recombination explicitly. This analysis uses a singular perturbation technique similar to that of Cohen and Kruskal ${ }^{3}$ and is omitted as it provides little additional information.

\section{FORMULATION OF THE BASIC EQUATIONS}

The equations below, except for the term $\epsilon n_{e} n_{i}$, are those used by Cohen and Kruskal $^{3}$ but are written in somewhat different dimensionless variables. They represent a quiescent, longitudinally uniform positive column with constant neutral and electron temperatures. For mathematical simplicity the cross section is taken with plane geometry:

$$
\begin{aligned}
& \frac{d J}{d y}=\gamma n_{e}-\epsilon n_{e} n_{i} \\
& \frac{d n_{e}}{d y}=-n_{e} E-\delta \tau J
\end{aligned}
$$

$$
\begin{gathered}
\frac{d n_{i}}{d y}=\tau n_{i} E-\tau J, \\
\frac{1}{\zeta} \frac{d E}{d y}=n_{i}-n_{e},
\end{gathered}
$$

where $J, n_{\epsilon}, n_{i}$, and $E$ are the dimensionless electron (and ion) flux, electron density, ion density, and electric field, to be determined for $0 \leq y \leq 1$ with the boundary conditions

$$
\begin{array}{ll}
y=0: J=0, & E=0, \quad n_{e}=1, \\
y=1: n_{e}=0, & n_{i}=0 .
\end{array}
$$

The variables above are related to the physical variables and coefficients by $y=\hat{x} / L, J=\left(L T_{W} \hat{\Gamma}\right) /$ $\left(D_{i} N_{e 0} T_{e}\right), n_{e}=N_{e} / N_{e 0}, n_{i}=N_{i} / N_{e 0}, E=$ $(e L \hat{E}) /\left(k T_{e}\right), \gamma=\left(L^{2} \nu_{I} T_{W}\right) /\left(D_{i} T_{e}\right), \epsilon=\left(L^{2} \alpha N_{e 0} T_{W}\right) /$ $\left(D_{i} T_{e}\right), \delta=D_{i} / D_{e}, \tau=T_{o} / T_{W}, \zeta=\left(N_{e 0} L^{2} e^{2}\right) /$ $\left(\epsilon_{0} k T_{e}\right)$, where $\hat{x}$ is the distance from the axis of the column, $\hat{\Gamma}$ is the flux of electrons and ions toward the wall, $\hat{E}$ is the "radial" electric field (in the $\hat{x}$ direction), $N_{e}$ and $N_{i}$ are the number densities of electrons and ions, $N_{e 0}=N_{\theta}(0), L$ is the distance from the axis to the wall, $T_{e}$ and $T_{W}$ are the electron and neutral temperatures, $D_{e}$ and $D_{i}$ are the electron and ion diffusion coefficients, $\nu_{I}$ and $\alpha$ are the ionization and recombination coefficients, and $e, k, \epsilon_{0}$ are the magnitude of the electron charge, the Boltzmann constant, and the permittivity of free space.

The boundary conditions $J=0, E=0$ at $y=0$ reflect the symmetry of the problem. The conditions $n_{e}=0, n_{i}=0$ at $y=1$ must be regarded as an approximation as discussed by McDaniel. ${ }^{6}$ The general question of the validity of the macroscopic equations near the wall is comprehensively discussed by Blank. ${ }^{4}$

For fixed density and temperature of the neutral gas, the quantities $T_{e}, \nu_{I}, D_{e}, D_{i}$, and $\alpha$ become functions of the axial electric field only. Hence, the coefficients $\gamma, \delta$, and $\tau$ are all functions of the $E_{z}$ whereas $\epsilon$ and $\zeta$ depend on both $E_{z}$ and $N_{e 0}$. Since there are five boundary conditions for four firstorder equations, the extra condition defines the relationship between $E_{\mathrm{z}}$ and $N_{e 0}$.

In the following analysis it is important to know the relative magnitudes of variables and parameters. As an example let us choose hydrogen gas at $300^{\circ} \mathrm{K}$ and $1 \mathrm{~mm} \mathrm{Hg}$ in a discharge with $L=1 \mathrm{~cm}$. The coefficient $\zeta$ is large because the electron density in glow discharges typically lies between $10^{8}$ and $10^{12} \mathrm{~cm}^{-3}$. Since recombination is only important at large densities, the calculations in this work are arbitrarily restricted to the range $10^{10} \mathrm{~cm}^{-3}<$ 
$N_{\mathrm{e} 0}<10^{12} \mathrm{~cm}^{-3}$. Data are available for $13.4 \mathrm{~V} / \mathrm{cm}<$ $E_{x}<32.2 \mathrm{~V} / \mathrm{cm}$, and these bounds on $N_{\mathrm{e} 0}$ and $E_{z}$ restrict the coefficients to the ranges, $0.0814<$ $\gamma<20.5,2.36 \times 10^{-4}<\delta<3.92 \times 10^{-4}, 73.5<$ $\tau<153,2.88 \times 10^{-2}<\delta \tau<3.60 \times 10^{-2}, 6.69 \times$ $10^{-3}<\epsilon<1.53,4.53 \times 10^{3}<\zeta<9.53 \times 10^{5}$. The choice of the dimensionless variables is such that their order of magnitude is expected to be unity throughout most of the discharge. The various terms can then be roughly estimated by the size of their coefficients.

\section{SIMPLIFICATION AND SOLUTION OF THE EQUATIONS}

The work of Cohen and Kruskal ${ }^{3}$ shows that for large $N_{\epsilon}$, the positive column consists of two regions in which the equations must be solved by different methods. The first is the main or central region, in which the term $(1 / \zeta)(d E / d y)$ can be neglected to lowest order with the result $n_{e}=n_{i}$ and a considerable simplification of the equations. The second region is a thin boundary layer or sheath near the wall where $(1 / \zeta)(d E / d y)$ is comparable in magnitude to the other terms. The solutions in the two regions are obtained in the form of asymptotic series in functions of $\zeta$, which contain undetermined parameters that are evaluated in a process of matching. The presence of the additional term $\epsilon n_{e} n_{i}$ does not alter the separation in main region and sheath, and an asymptotic solution for the Eqs. (1)-(4) has been obtained. However, the effect of the term $(1 / \zeta)$. $(d E / d y)$ is of lesser importance at large electron densities where recombination is significant, and hence the following analysis is restricted to the approximation $n_{e}=n_{i}$.

The equations and boundary conditions (1)-(6) now become, after some manipulations,

$$
\begin{gathered}
\frac{d J}{d y}=\gamma n_{e}-\epsilon n_{e}^{2}, \\
\frac{d n_{e}}{d y}+\tau \frac{1+\delta \tau}{1+\tau} J=0, \\
\frac{d n_{e}}{d y}+\frac{1+\delta \tau}{1-\delta} n_{e} E=0, \\
y=0: \quad J=0, \quad E=0, \quad n_{e}=1,
\end{gathered}
$$

$$
y=1: n_{s}=0 .
$$

By eliminating $J$ between Eqs. (7) and (8) there is obtained

$$
\frac{d^{2} n_{e}}{d y^{2}}+\gamma^{*} n_{e}-\epsilon^{*} n_{e}^{2}=0
$$

where

$$
\gamma^{*}=\gamma \tau \frac{1+\delta \tau}{1+\tau}, \quad \epsilon^{*}=\epsilon \tau \frac{1+\delta \tau}{1+\tau} .
$$

The boundary conditions are

$$
\begin{aligned}
& y=0: n_{\epsilon}=1, \quad \frac{d n_{\epsilon}}{d y}=0, \\
& y=1: n_{\epsilon}=0,
\end{aligned}
$$

where the second of Eq. (13) follows from Eqs. (9) and (10). Equations (12)-(14) serve to determine $\gamma^{*}$ as a function of $\epsilon^{*}$ and $n_{e}$ as a function of $y$ and $\epsilon^{*}$. Once $n_{\bullet}$ is found, $J$ and $E$ can be obtained from Eqs. (8) and (9).

Except for the recombination term, Eq. (12) is the same as the standard ambipolar diffusion equation' as can be seen by expressing its coefficients in terms of the original dimensional quantities. Using the definitions of $\delta, \tau$, etc., and the Einstein relations ${ }^{2}$ for the mobilities $D_{o} / \mu_{e}=k T_{c} / e, D_{i} / \mu_{i}=k T_{W} / e$ there is obtained

$$
\begin{gathered}
\frac{1+\delta \tau}{1+\tau}=\frac{D_{i}}{D_{a}}, \\
\gamma^{*}=\gamma \tau \frac{1+\delta \tau}{1+\tau}=\frac{L^{2} \nu_{I}}{D_{a}}, \\
\epsilon^{*}=\epsilon \tau \frac{1+\delta \tau}{1+\tau}=\frac{L^{2} \alpha N_{\varepsilon 0}}{D_{a}},
\end{gathered}
$$

where

$$
D_{a}=\frac{\mu_{e} D_{i}+\mu_{i} D_{e}}{\mu_{e}+\mu_{i}}
$$

is the ambipolar diffusion coefficient.

The solution of Eqs. (12)-(14) can be reduced to quadrature by a standard substitution, using $n$ as the independent and $d n / d y$ as the dependent variable. The resulting integrals can be expressed in terms of elliptic functions as

$$
\begin{gathered}
n=\frac{1-[(1-a+b)(-1+3 a+b) / 4 a b] \mathrm{sn}^{2}\left[\gamma(b)^{1 / 2} y / 2, k\right]}{1-[(-1+3 a+b) / 2 b] \mathrm{sn}^{2}\left[(\gamma b)^{1 / 2} y / 2, k\right]}, \\
\gamma^{* 1 / 2}=\frac{2}{b^{1 / 2}} \int_{0}^{A} \frac{d z}{\left[\left(1-z^{2}\right)\left(1-k^{2} z^{2}\right)\right]^{1 / 2}}, \quad(16) \quad a=\frac{2}{3} \frac{\epsilon^{*}}{\gamma^{*}}, \quad b=\left(1+2 a-3 a^{2}\right)^{1 / 2},
\end{gathered}
$$




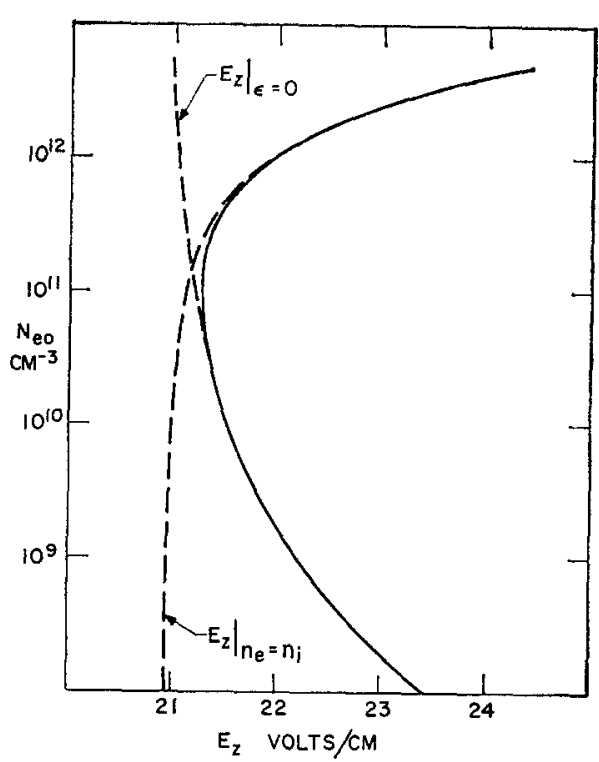

FIg. 3. The $E_{z}-N_{e 0}$ curve including recombination.

$$
\begin{aligned}
k & =\left(\frac{3 a-1+b}{2 b}\right)^{1 / 2} \\
A & =\left(\frac{4 a b}{(1-a+b)(-1+3 a+b)}\right)^{1 / 2}
\end{aligned}
$$

and $x=\operatorname{sn}(u, k)$ is defined as the solution of

$$
\int_{0}^{x} \frac{d z}{\left[\left(1-z^{2}\right)\left(1-k^{2} z^{2}\right)\right]^{1 / 2}}=u \text {. }
$$

The solution can also be obtained in powers of $\epsilon^{*}$ by introducing

$$
\begin{aligned}
& n_{e}=n_{0}+\epsilon^{*} n_{1}+\epsilon^{*^{2}} n_{2}+\cdots, \\
& \gamma^{*}=\gamma_{0}^{*}+\epsilon^{*} \gamma_{1}^{*}+\epsilon^{*^{2}} \gamma_{2}^{*}+\cdots
\end{aligned}
$$

in Eqs. (12)-(14) and solving the resulting linear problems. The expansion has been evaluated up to the $\epsilon^{*^{2}}$ term for $\gamma^{*}$ :

$$
\gamma^{*} \cong \frac{\pi^{2}}{4}+\frac{8}{3 \pi} \epsilon^{*}+\left(\frac{10}{3 \pi^{2}}-\frac{32}{9 \pi^{3}}-\frac{64}{3 \pi^{4}}\right) \epsilon^{*^{2}} \text {. }
$$

For $\epsilon^{*}<11$, Eq. (21) is in error by less than $0.5 \%$ compared to the exact expression. A power series similar to Eq. (18) can be obtained for a cylindrical discharge where a closed form solution is not possible.

\section{RESULTS AND DISCUSSION}

Figure 3 presents typical $E_{\mathrm{z}}-N_{\mathrm{e} 0}$ characteristics. The dashed curve for $E_{z}$ is obtained from Eq. (16), and the curve indicated by $\left.E_{2}\right|_{6=0}$ represents the solution of Eqs. (1)-(6) with $\epsilon=0$, i.e., without recombination as obtained by the singular perturbation technique of Cohen and Kruskal. ${ }^{3}$ A similar singular perturbation has been applied by the present authors to the complete Eqs. (1)-(6) and the solution is presented by the full curve of Fig. 3 . It is seen that for large $N_{e 0}$ where recombination is important the simplified solution presented in the preceding section is adequate. The importance of recombination depends on the magnitude of the coefficient $\alpha$, a quantity that is very difficult to measure due to its dependence on impurities. Figure 3 has been obtained with $\alpha=3 \times 10^{-8} \mathrm{~cm}^{3} \mathrm{sec}^{-1}$, which is considered as an upper bound for the recombination coefficient in pure hydrogen. ${ }^{7}$ Higher values of $\alpha$ have been observed in inert gases ${ }^{8}$ and in hydrogen with impurities, ${ }^{7.8}$ although in these cases the recombination mechanism is different. In Fig. 3, homogeneous recombination becomes important at electron densities of about $10^{12} \mathrm{~cm}^{-3}$, i.e., near the upper limit of the glow discharge. For higher values of $\alpha$ or $L$, recombination becomes important at lower electron densities. For lower values of $\alpha$ or $L$, recombination becomes important at higher electron densities, where however, our basic equations may cease to apply.

As in previous studies ${ }^{2-4}$ we have considered electrons and a single positive ion species. This is appropriate for discharges in molecular gases such as $\mathrm{H}_{2}$ and $\mathrm{N}_{2}$. In $\mathrm{He}$ or Ar discharges, atomic and molecular ions are both present. The atomic ions, formed by electron impact, react to give molecular ions according to

$$
\mathrm{Ar}^{+}+\mathrm{Ar}+\mathrm{Ar} \rightarrow \mathrm{Ar}_{2}^{+}+\mathrm{Ar}
$$

The molecular ions formed are mainly responsible for the recombination by the dissociative mechanism

$$
\mathrm{Ar}_{2}^{+}+e \rightarrow 2 \text { Ar. }
$$

This two ion system is expected to have an $E_{\mathrm{z}}-N_{\mathrm{e} 0}$ curve similar to that of Fig. 3 but a quantitative analysis must, in general, include conservation equations for both molecular species. A similar complication arises in discharges with negative ions.

In addition to recombination, some other nonlinear effects may be important in shaping the $E_{z}-N_{e 0}$ curve for large values of $N_{e 0}$. One such effect is due to the increase in neutral temperature as $N_{e 0}$ increases. The resulting higher temperature at the center causes a lower neutral density and a higher ionization there. Thus, the electron loss to the walls can be balanced by a lower $E_{z}$. This effect, which will cause the curve of Fig. 3 to eventually bend to the left, may be concommitant to the glow-to-arc transition in low-pressure discharges. In high-pressure discharges, at high currents the neutral temperature approaches the 
electron temperature and our basic equations (1)-(4) are no longer applicable.

\section{ACKNOWLEDGMENT}

The first author has been supported by a Hertz Foundation Fellowship.

${ }^{1}$ A. Von Engel, Ionized Gases (Oxford University Press, London, 1965), Chap. 8.
${ }^{2}$ W. P. Allis and D. J. Rose, Phys. Rev. 93, 84 (1954).

${ }^{3}$ I. M. Cohen and M. D. Kruskal, Phys. Fluids 8, 920 (1965).

${ }^{4}$ J. L. Blank, Phys. Fluids 11, 1686 (1968).

5 J. D. Cobine, Gaseous Conductors (Dover, New York, 1958), Chap. VIII.

- E. W. McDaniel, Collision Phenomena in Ionized Gases (Wiley, New York, 1964), p. 496.

7 K. B. Persson and S. C. Brown, Phys. Rev. 75, 729 (1955).

${ }^{8}$ D. R. Bates and A. Dalgarno, in Atomic and Molecular Processes, edited by D. R. Bates (Academic, London, 1962), p. 267.

\title{
Hydromagnetic Oscillations of a Tangential Discontinuity in the Chew, Goldberger, and Low Approximation
}

\author{
S. Duhad, F. Gratton, and J. Gratton \\ Departamento de Física, Facultad de Ciencias Exactas y Naturales, Universidad de Buenos Aires \\ Buenos Aires, Argentina \\ (Received 2 December 1968; final manuscript received 15 December 1969)
}

\begin{abstract}
The differential equation for linear modes of oscillation of plane parallel flows of plasmas along an external magnetic field in the Chew, Goldberger, and Low approximation is obtained. Properties of modes for a tangential discontinuity are studied for the case when the surface is modulated along the magnetic field. Overstable modes found by other authors are shown to be spurious. Regions of existence of modes, proper frequencies, and spatial dependence of the perturbation are given. It is found that, broadly speaking, low $\beta$ plasmas should be free of surface instabilities for all values of the flow velocity, whereas high $\beta$ plasmas can be unstable if the flow velocity is nearly sonic. Changes in the anisotropy do not substantially affect the general picture of the problem.
\end{abstract}

\section{INTRODUCTION}

The problem of the stability of parallel flows of plasmas has attracted some interest both theoretical and experimental in recent years. D'Angelo and von Goeler ${ }^{1,2}$ have performed experiments on the instabilities of plasmas with velocity gradients and have shown its connection with anomalous diffusion. $^{3}$

The case of a tangential discontinuity has been studied theoretically in magnetohydrodynamics (a review can be found in Ref. 4), and in the electrostatic approximation for a collisionless plasma $^{5-9}$; in the Chew, Goldberger, and Low approximation, ${ }^{10}$ it has been studied by Talwar ${ }^{11}$ who has suggested that calculations with a collisionless anisotropic model may be of interest in a variety of astro- and space-physics situations.

However, the detailed properties of the hydromagnetic Chew, Goldberger, and Low modes have not been studied, and our analysis discloses that some of the solutions obtained in Ref. 11 are spurious; among these, the overstable modes found by Talwar ${ }^{11}$ are spurious as we shall presently show.
In this paper we propose, first, to derive the differential equation for the oscillations in parallel flows of plasma in the Chew, Goldberger, and Low approximation, and second, to analyze in detail the case of a discontinuity surface in the velocities.

\section{DIFFERENTIAL EQUATION AND PARAMETERS OF THE PROBLEM}

In the unperturbed state, we shall consider a plasma flowing in the $x$ direction with velocity $u=u(y)$ and density $\rho=\rho(y)$. The magnetic field $\mathbf{B}_{0}$ is uniform and parallel to the flow. We shall assume that $p_{\|}$and $p_{\perp}$, the components of the stress tensor along, and normal to the magnetic field $\mathbf{B}_{0}$ are constant.

We shall consider small departures from this stationary state and linearize the equations of Chew-Goldberger-Low. ${ }^{10}$ As is well known, this approximation applies to a dilute plasma in a strong magnetic field when the heat flow is negligible, and the frequency of the perturbation is much less than the ion gyrofrequency.

We Fourier analyze any perturbed quantity $Q$ 